\title{
Effective Health Care bulletins: are they efficient?
}

\author{
David Torgerson, Mandy Ryan, Cam Donaldson
}

In 1992 the Department of Health commissioned a series of bulletins on effectiveness since evidence was available on chosen healthcare interventions. From this project eight Effective Health Care bulletins have been produced on a range of topics (box). Edited versions have been published in the journal. This paper and the following paper comprise an unsolicited critique of the Effective Health Care bulletins and a response by the authors of the bulletins.

\begin{tabular}{|l|}
\hline Effective Health Care Bulletins \\
1992 \\
No 1-Screening for Osteoporosis to Prevent \\
Fractures \\
No 2-Stroke Rehabilitation \\
No 3-Management of Subfertility \\
No 4-Treatment of Persistent Glue Ear in \\
Children \\
1993 - \\
No 5-Treatment of Depression in Primary \\
Care \\
No 6-Cholesterol: Screening and Treatment \\
No 7-Brief Interventions and Alcohol Use \\
1994 \\
No 8-Implementing Clinical Practice \\
Guidelines
\end{tabular}

A key factor in securing a more effective and efficient healthcare service is the availability of valid effectiveness and efficiency data. Such data provision is the stated objective of the series of Effective Health Care bulletins, which is "to provide valid and systematic data to aid managers and clinicians in purchasing and providing healthcare services." 1

In this paper we question the extent to which the information in such bulletins will aid clinicians and managers in making decisions about the efficient allocation of scarce healthcare resources, and we suggest improvements to the bulletins such that they can become a better tool for helping healthcare purchasers set priorities and place contracts.

The first section comprises an overview of the bulletins and examines the three criteria that the effective health care team producing the bulletins propose as aids to managers and clinicians involved in purchasing and providing in the NHS: clinical effectiveness, cost effectiveness, and acceptability. A fourth criterion is also examined. This criterion is implicitly recognised by the team as being important for identifying topics for bulletins and purchasing decisions: burden (or cost) of illness considerations. The team recommend alternative interventions for purchasers to consider. The basis on which these alternatives are suggested is also questioned. We also argue that, to aid managers and clinicians, questions posed by the team need to be within a marginal context as this represents the reality of healthcare decision making.

\section{Effective Health Care bulletins: overview} CLINICAL EFFECTIVENESS

The first criterion that the team argue is important is the clinical effectiveness of treatment. ${ }^{1}$ Clearly, this is so; however, what is not quite so clear is the definition of "effectiveness." The bulletins concentrate on clinical effectiveness - for example, in the bulletin on the management of infertility "live birth" was taken as the measure of effectiveness, so allowing computation of "cost per live birth" as a measure of efficiency. ${ }^{2}$ However, there may be other benefits to consider beyond this narrow definition - for example, in evaluating infertility treatment important outcomes may include coming to terms with infertility and knowing that everything possible has been done to have a child. ${ }^{3}$ Such outcomes were simply acknowledged but not taken account of by the team.

The important question that emerges is what measure of effectiveness is relevant to purchasers. If purchasers are concerned solely with clinical effectiveness then the approach taken by the team is justified. However, if purchasers are concerned with what is important to users of health services, as recent government documents have suggested they should be, then the clinical effectiveness approach may not be justified.

\section{COST EFFECTIVENESS}

The team proposed the use of the economic technique of cost effectiveness analysis for the bulletins. The context of the bulletins is such that only a narrow range of healthcare interventions is considered, and there is also a need to avoid the bulletins being overly complex. Therefore, the choice is probably correct. However, such a technique can answer only the question of which is the most cost effective method of providing a service. Cost effectiveness analysis can take two forms. Firstly, if alternatives have been shown to produce the same amounts of health outcome, cost effectiveness analysis would entail looking at the least costly way of producing the service. Secondly, if outcomes are different and a fixed budget is available the alternative which minimises cost per unit of health gain is more cost effective. For example, in the case of the bulletin on managing infertility it seems implicit that a decision has been taken to carry out infertility treatment, and the important question is what type of treatment gives rise to the lowest cost per live birth. 
However, cost effectiveness analysis cannot answer the broader question of whether purchasers should buy, for example, more or less infertility treatments, or whether they should purchase osteoporosis screening at all. The relevant economic framework for answering such questions is cost-benefit analysis, in which the allocation of more resources to infertility treatments or osteoporosis screening programmes would have to be weighed against the benefits given up by taking these resources from another part of the healthcare system. Yet information in the bulletins has not been put in this context. Indeed, the potential costs of establishing an osteoporosis screening service were described but were not related to benefits.

Instead, within the context of the first bulletin (screening for osteoporosis ${ }^{1}$ ), a cost effectiveness approach might be argued to be appropriate since the assumption was that purchasers had decided that attempts should be made to reduce fractures of the hip, wrist, and spine in elderly women. However, by not relating costs to effectiveness the first bulletin also failed to carry out a cost effectiveness analysis. Furthermore, although the main policy question of that bulletin was "whether to screen," this question is inseparable from evaluation of treatment as, clearly, screening should not be undertaken without a viable intervention. Failure by the team to undertake a cost effectiveness analysis, however crude, means that purchasers cannot judge whether prevention of osteoporosis using alternatives to hormone replacement therapy, such as calcium supplementation, etidronate, calcitonin, and Vitamin D, are more or less cost effective than hormone replacement therapy, with or without screening.

\section{ACCEPTABILITY}

The team consider that acceptability of a healthcare intervention is an important criterion in deciding on provision, arguing that, even if a healthcare intervention is clinically effective and economically cost effective, if its acceptability is low, then its provision must be open to doubt. By implication, therefore, the acceptability of a healthcare intervention would seem to be a more important criterion than clinical effectiveness and cost effectiveness. However, before issues of acceptability are considered we need to define what is meant by the term.

In the first bulletin the team seemed to regard acceptability as synonymous with compliance or uptake of screening for osteoporosis. ${ }^{1}$ Therefore, the higher the uptake by the target population the more acceptable the healthcare intervention. In that bulletin it was implied that screening uptake must exceed $72 \%$ for the intervention to be an acceptable. Furthermore, it was argued that if a healthcare intervention has poor compliance (acceptability) then ways of enhancing its acceptability must be considered. However, increasing compliance with screening programmes is often associated with increased costs. It has been argued elsewhere that increasing uptake or compliance with screening may not be an efficient allocation of scarce healthcare resources. ${ }^{4}$ For example, if $90 \%$ compliance produces a fall in the target disease of $30 \%$ (as it does for breast screening ${ }^{5}$ ) should a screening programme not be implemented because it is acceptable only to $45 \%$ of the population and so produces only a $15 \%$ fall in disease, but at half the cost? If a screening programme, or any other health programme, is judged purely in efficiency terms - that is, cost per unit of health benefit generated - then a healthcare intervention can be judged efficient whatever the uptake rate. However, if the acceptability criterion is used, then a healthcare intervention that can efficiently prevent significant morbidity and mortality might be rejected by purchasers.

We argue that if a healthcare intervention fails either the effectiveness or cost effectiveness criteria, then it is reasonable to recommend against its purchase. In contrast, we assert that if a healthcare intervention were clinically effective and relatively cost effective, but failed to be acceptable to most of the population, then it could (and probably should) still be purchased if the size of the programme can be tailored to the level of acceptability.

Acceptability may be important, in economic terms, for certain individual healthcare interventions for two reasons. Firstly, if those who find a healthcare intervention unacceptable have the greatest ability to benefit then investing resources to improve acceptability may be worthwhile. Secondly, poor acceptability might affect the efficiency of a healthcare intervention if it is necessary to realise economies of scale. For example, all healthcare interventions will incur certain start up costs. For relatively uncommon conditions high uptake by the target population may be required to ensure sufficient throughput to justify these start up costs. In addition, for certain healthcare procedures, particularly surgery, effectiveness will only be maximised if surgeons treat a minimum number of patients in order for them to maintain their surgical skill, and this applies to managing infertility, where it was argued by the team that at least 750 treatments of in vitro fertilisation are required annually to justify the initial start up costs. $^{2}$ However, "acceptability" was not addressed explicitly in this bulletin.

Ethically, it could be argued that a healthcare programme should still be provided, despite its unacceptability, to certain population groups. For instance, some tests for foetal abnormalities, such as amniocentesis, are unpleasant to the patient and are clearly unacceptable to many women. However, if an individual woman is risk averse to bearing a child with Down syndrome and wishes to undergo such a test should the opportunity be denied only on the grounds that many other women find it unacceptable?

In summary, there may be some interventions for which high uptake is justified in economic terms. However, it is not necessary to use a blanket criterion of acceptability for all 
health interventions. Acceptability is useful only in so far as it contributes to costs and benefits.

IDENTIFYING AND SETTING PRIORITIES: COST OF ILLNESS AND BURDEN OF SUFFERING

The team do not make clear how topics are identified for the bulletins, ${ }^{1}$ although it seems that they are chosen if, among other things, the intervention has high resource implications, ${ }^{1}$ or imposes a large burden of suffering on the population. For example, in the bulletins on screening for osteoporosis, ${ }^{1}$ cholesterol screening, ${ }^{6}$ and rehabilitation of stroke the suggestion is that because osteoporosis and cardiovascular disease pose major health burdens they should be candidates for intervention. This approach of assessing or adopting a healthcare intervention is firmly rooted in the total needs or cost of illness approach to priority setting, which was previously criticised as unhelpful and misleading. ${ }^{7-9}$

Burden of illness is, admittedly, only one criterion used by the team on which to base purchasing decisions, as evidenced by the conclusion in the first bulletin that screening for osteoporosis is not a "good buy," despite it satisfying the burden of illness criterion. The potential danger of using burden of illness both to identify and set priorities is that diseases associated with highly effective and low cost interventions but that do not contribute significantly to the overall burden of suffering would not be considered by the team. In economic terms, whether a disease contributes a small or large amount to the overall burden of suffering is not an issue. What is important is setting the benefits of any intervention against their costs. For instance, phenylketonuria does not contribute greatly to the overall burden of suffering whether the entire population is chosen as the denominator or only children born with some form of congenital disease. However, testing and intervention are highly effective relative to cost, and therefore screening for this disease is, and should be, undertaken.

A referee to this journal suggested that, all things being equal, including cost effectiveness, then it would be fair to consider burden of illness issues. However, if the same number of people could be cured at the same cost but one group had a rare condition and the other a common ailment then it would seem to be arbitrary (perhaps inequitable) to base decisions of resource allocation on whether or not a disease is common or rare when health gain per pound spent is equal.

\section{ALTERNATIVE INTERVENTIONS}

Although the objective of the bulletins is to focus on one issue, such as bone density screening, it would be worthwhile to purchasers to know of any potential alternatives for treating or preventing a condition. However, it must be made clear whether potential alternatives are supported by existing evidence, otherwise purchasers might invest in potentially ineffective and costly alternatives.
For example, in the first bulletin minimising external hazards was recommended as an alternative option in preventing osteoporotic fractures. This intervention would presumably need some form of audit of all elderly people's houses to identify hazards likely to cause falls, probably itself an extremely expensive exercise without the additional cost implications of correcting identified hazards. Indeed, a recent randomised trial has failed to show that falls can be prevented. ${ }^{10}$

The team also recommended that health authorities investigate the use of population strategies of preventing not only osteoporosis but coronary heart disease. ${ }^{11}$ Although the population approach to prevention is attractive, purchasing authorities should be cautious in that this approach probably requires further research before it can be deemed effective or cost effective. For example, in a 20 year follow up of a smoking cessation trial it was noted that in the nonintervention group smoking cessation was very high and therefore additional intervention conferred only a modest, statistically nonsignificant, marginal benefit. ${ }^{12}$ A similar criticism of population prevention may apply to advocacy for interventions to reduce alcohol consumption and serum cholesterol concentrations.

\section{What do purchasers need?}

As recognised by the team, the starting point for the bulletins should be to specify the question that policymakers face. Within the context of current decision making, these purchasing questions are likely to be at the margin. This margin may be a change in the current level of services, or whether to provide a service at all. For example, in the case of osteoporosis, for those districts that do not have a contract for osteoporosis screening the relevant margin is whether to provide screening programmes. However, most districts will have some level of infertility treatment, even if this service comprises only infertility drug treatment. Thus, rather than whether or not to purchase a comprehensive infertility service (the question posed in the bulletin on managing infertility ${ }^{2}$ ), the two questions most purchasers face for providing infertility services are either how to reallocate existing resources to increase efficiency or how to allocate some additional sum of money to infertility services. With regard to the first question, one possible "margin" to examine would be women who are currently offered tubal surgery who would have a better chance of leaving the infertility service with a child if they went through in vitro fertilisation. Thus, cost per live birth could be reduced if resources were reallocated such that some women currently offered tubal surgery were instead offered in vitro fertilisation. A given amount of live births could be produced at less cost or more live births could be produced for a fixed sum. With regard to the second question, this would decide what proportion of money to give to specialist services (in vitro fertilisation and gamete intrafallopian transfer) versus more traditional 
treatment, and who should have access to such services. These are again decisions at the margin. The second question involves the situation in which an intervention is more costly and more effective, resulting in the possibility of resources being taken from other areas of healthcare to facilitate its implementation. All that the bulletins can be used for in this situation is to provide information on costs and benefits for health authorities or boards to use in local situations to decide whether such an intervention should be funded.

The bulletins, to an extent, address the first question but rarely the second. When the second question has been addressed, this has not been done so in a realistic context. For instance, rather than analysing the cost of establishing a subfertility service, surely it would be more relevant to examine the costs and benefits of expanding (or contracting) that service. A marginal context (that is, looking at costs and benefits of changes in levels of service) would be more relevant for the bulletins.

Furthermore, acceptability and burden of illness are relevant not on their own but only in so far as they affect these two questions. With regard to alternative interventions, these should be suggested only for those alternatives for which there is solid evidence of their effectiveness and cost effectiveness.

\section{Conclusion}

The effective health care team has made an admirable attempt to provide purchasers with information to aid them in priority setting. Effectiveness and cost effectiveness play a part in this, but not necessarily acceptability or burden of illness. We suggest that the information in the bulletins should be presented according to the types of question that policymakers may ask.

The Health Economics Research Unit is supported by the Chief Scientist Office of the Scottish Office Home and Health Scientist Office of the Scottish Office Home and Health Department. The views expressed are those

1 Effective Health Care. Screening for osteoporosis to preven fractures. Leeds: School of Public Health, University of Leeds; Centre for Health Economics, University of York; Research Unit, Royal College of Physicians, 1992 (Bulletin No 1.)

2 Effective Health Care. The management of subfertility. Leeds: School of Public Health, University of Leeds; Centre for Health Economics, University of York; Research Unit, Royal College of Physicians, 1992. (Bulletin No 3.)

3 Ryan M. Economic evaluation if in vitro fertilisation: examining the benefits. Aberdeen: University of Aberdeen, 1992. (HERU Discussion paper 13/92.)

4 Torgerson DJ, Donaldson C. An economic view of high compliance as a screening objective. BMF 1994;308 $17-9$.

5 Tabar L, Fagerberg G, Duffy SW, Day NE. The Swedish two county trial of mammographic screening for breast cancer: recent results and calculation of benefit. Epidemiol Community Health 1988;43:107-14.

6 Effective Health Care. Cholesterol: screening and treatment. Leeds: Institute for Health, University of Leeds; Centr for Health Economics, University of York; Research Unit, Royal College of Physicians, 1993. (Bulletin No 6.)

7 Sheill A, Gerard K, Donaldson C. Cost of illness studies: an aid to decision making? Health Policy 1987;10:317-23.

8 Donaldson C, Mooney G. Needs assessment, priority setting, and contracts for health care: an economic view. BMF 1991;303:1529-30.

9 Mooney G, Healey A. Strategy full of good intentions. BMF 1991:303:1119-20.

10 Vetter NJ, Lewis PA, Ford D. Can health visitors prevent fractures in elderly people? BMF 1992;304:888-90.

11 Effective Health Care. Brief interventions and alcohol use. Leeds: Institute of Health, University of Leeds; Centre for Leeds: Institute of Health, University of Leeds; Centre for Health Economics, University of York; Research

12 Rose G, Colwell L. A randomised trial of anti-smoking advice: 20 year results. $\mathcal{f}$ Epidemiol Community Health 1992;46:75-7. 